\title{
Wie können wir die Ursachen sozialer Ungleichheit verstehen?
}

Martin Diewald, Rainer Riemann

5.1 Einleitung -68

Birgit Spinath

5.2 Interview mit Prof. Martin Diewald, Professor für Soziologie an der Universität Bielefeld, und Prof. Rainer Riemann, Professor für Psychologie an der Universität Bielefeld - 68

Zitierte und weiterführende Literatur - 78 


\section{1 $\quad$ Einleitung}

Birgit Spinath

Soziale Ungleichheit ist aufgrund der wachsenden Unterschiede zwischen Bevölkerungsgruppen als gesellschaftliches Problem aktueller denn je. Ungleichheit muss per se noch kein Problem sein. Jedoch kann aus Ungleichheit leicht Ungerechtigkeit werden, wenn es etwa um Bildungschancen und soziale Partizipation geht. Daher ist soziale Ungleichheit eines der klassischen Forschungsfelder der Soziologie. Auch die Psychologie beschäftigt sich mit Ungleichheit. Insbesondere die Differenzielle Psychologie macht es sich zur Aufgabe, die Art, das Ausmaß und die Entstehung von individuellen Unterschieden zu verstehen. Dazu nutzt sie unter anderem die Methode der Verhaltensgenetik, durch die festgestellt werden kann, in welchem Ausmaß sich interindividuelle Unterschiede auf unterschiedliche genetische Ausstattung zurückführen lassen. Tatsächlich ist inzwischen für wichtige psychische Merkmale wie Intelligenz, Persönlichkeit und Motivation eine erhebliche genetische Mitbedingtheit nachgewiesen worden. Um die Ursachen sozialer Ungleichheit verstehen zu können, müssen diese individuellen Unterschiede und ihre Entstehung mit berücksichtigt werden. Zu diesem Zweck ist eine transdisziplinäre Zusammenarbeit von Soziologie und Psychologie erforderlich.

Prof. Martin Diewald ist Soziologe und hat eine Professur für Sozialstrukturanalyse inne. Eines seiner zentralen Forschungsthemen ist soziale Ungleichheit (z. B. Diewald, 2010a, b). Prof. Rainer Riemann ist Psychologe und hat eine Professur für Differenzielle Psychologie, Persönlichkeitspsychologie und Psychologische Diagnostik inne. Eines seiner zentralen Forschungsthemen ist die Verhaltensgenetik (Riemann und Spinath, 2005). Seit 2012 haben die beiden ein gemeinsames, groß angelegtes Forschungsprojekt (mit Prof. Frank M. Spinath), in dem sie die Ursachen sozialer Ungleichheit unter Berücksichtigung genetischer Informationen untersuchen. Aus dieser transdisziplinären Zusammenarbeit werden weitreichende neue Erkenntnisse erwartet.

\section{Referenzen}

Diewald, M. (2010a). Ungleiche Verteilungen und ungleiche Chancen. Zur Entwicklung sozialer Ungleichheiten in der Bundesrepublik. In F. Faulbaum \& C. Wolf (Hrsg.), Gesellschaftliche Entwicklungen im Spiegel der empirischen Sozialforschung. Schriftenreihe der ASI-Arbeitsgemeinschaft Sozialwissenschaftlicher Institute (S. 11-38). Wiesbaden: VS Verlag für Sozialwissenschaften.

Diewald, M. (2010b). Zur Bedeutung genetischer Variation für die soziologische Ungleichheitsforschung. Zeitschrift für Soziologie, 39, 4-21.

Riemann, R. (2008). Können wir unser Verhalten steuern? Verhaltensgenetische Aspekte. In R. Kögerler, F. Gruber \& M. Dürnberger (Hrsg.), Homo animal materiale. Die materielle Bestimmtheit des Menschen (S. 41-64). Linz: Wagner.

Riemann, R., \& Spinath, F. (2005). Genetik und Persönlichkeit. In P. Netter \& J. Henning (Hrsg.), Biopsychologische Grundlagen der Persönlichkeit (S. 539-628). Heidelberg: Springer.

\subsection{Interview mit Prof. Martin Diewald, Professor für Soziologie an der Universität Bielefeld, und Prof. Rainer Riemann, Professor für Psychologie an der Universität Bielefeld}

Das Interview führten Clara Rücker, Luna Sabastian und Regina Sticker im Juni 2012. 
Interviewerin: Prof. Diewald, Sie sind Professor für Soziologie und beschäftigen sich schon seit längerer Zeit mit dem Thema »Soziale Ungleichheit«. Wann haben Sie angefangen, sich für das Thema zu interessieren?

Prof. Martin Diewald: Als Soziologe fängt man mit dem Studium an, sich mit Ungleichheit zu beschäftigen, einfach deswegen, weil sie eine der zentralen Gegenstände und Perspektiven in der Soziologie insgesamt ist: Dies betrifft zum einen die Erklärung sozialer Ungleichheiten, zum anderen die Frage, inwiefern Ungleichheiten selbst erklärend sind für etwas anderes, was uns Soziologen interessiert, beispielsweise soziale Integration. Als zentraler Forschungsgegenstand beschäftigt mich soziale Ungleichheit jetzt seit etwa drei bis vier Jahren.

I: Prof. Riemann, Sie sind Professor für Differenzielle Psychologie, ebenfalls an der Universität Bielefeld. Wie kommt es, dass Sie sich mit sozialer Ungleichheit befassen? Wie kam es zu der Kooperation mit Prof. Diewald?

Prof. Rainer Riemann: Differenzielle Psychologie beschäftigt sich mit den Unterschieden zwischen Menschen. Soziale Unterschiede zwischen Menschen oder soziale Ungleichheit sind natürlich ganz wichtige - aus meiner Perspektive schon sehr angewandte - Aspekte in der Differenziellen Psychologie. Von daher liegt es nahe, dass mir dieses Thema vertraut ist. Die Differenzielle Psychologie hat beispielsweise in der Intelligenzforschung einen engen Bezug zur Ungleichheitsforschung. Zur Kooperation ist es gekommen, weil Herr Diewald mich auf verhaltensgenetische Forschung zu sozialer Ungleichheit angesprochen hat. Sein Interesse fand ich so überraschend für einen Soziologen, dass ich mich dem nicht verschließen konnte.

\section{I: Wie definieren Sie soziale Ungleichheit?}

MD: Allgemein ist das die Besser- oder Schlechterstellung von Menschen in Relation zu anderen innerhalb einer Gesellschaft. Zu der Frage, was diese Besser- oder Schlechterstellung ausmacht, gibt es verschiedene Lösungsansätze: Bei ressourcentheoretischen Ansätzen geht es um die zentralen Ressourcen, von denen man annimmt, dass sie vielfältig eingesetzt werden können, um das, was einem individuell wichtig ist, zu erreichen: In unseren Gesellschaften ist das Geld, aber auch Macht und Ansehen. Außerdem gibt es ergebnisbezogene Ungleichheitskonzepte, bei denen es um Wohlfahrtspositionen und Verwirklichungschancen in verschiedenen Lebensbereichen geht.

I: Ist Ungleichheit gleichbedeutend mit Ungerechtigkeit? Falls nicht: Wann wird aus Ungleichheit Ungerechtigkeit?

MD: Auf keinen Fall haben beide Begriffe die gleiche Bedeutung.

Ungerechtigkeit entsteht, wenn die bestehenden Ungleichheiten - das können sowohl Ergebnisungleichheiten sein, also das Ausmaß der Ungleichheit insbesondere des Einkommens, oder (das ist im Allgemeinen diffiziler) Chancenungleichheiten - im Widerspruch zu individuellen und gesellschaftlichen Gerechtigkeitsvorstellungen stehen. Ein absolutes Maß für Gerechtigkeit gibt es nicht: Was als gerecht oder ungerecht gilt, ist Gegenstand und Ergebnis eines Diskurses. Es ist keineswegs so, dass alle Menschen die gleichen Vorstellungen haben, was gerecht oder ungerecht ist. Das unterscheidet sich innerhalb einer Gesellschaft, und das unterscheidet sich zwischen Gesellschaften: Amerikaner beispielsweise würden dabei andere Dinge in den Vordergrund stellen als wir. 
I: Das heißt, dass soziale Ungleichheit erst einmal ein nichtwertender Begriff ist und erst durch Gerechtigkeitsvorstellungen zu Ungerechtigkeit wird?

MD: Genau.

I: Wie misst man soziale Ungleichheit?

MD: Das hängt von den verschiedenen theoretischen Ansätzen ab, die man verfolgt. Man kann soziale Ungleichheit über die zentralen Ressourcen messen. Dann ist man bei der Eliteforschung, Einkommensforschung, Statuserwerbsforschung, wo Ungleichheit an dem Innehaben von Macht festgemacht wird oder - was durchaus etwas anderes ist - an Autonomiespielräumen, an der Selbstbestimmtheit. Inwiefern bin ich unabhängig davon, was andere mir darüber sagen, was ich zu tun und zu lassen habe? Oder an Einkommen und Vermögen als zentralen materiellen Ressourcen oder auch an Status als Summe von Prestige und Ansehens- bzw. Anerkennungspositionen innerhalb einer Gesellschaft, wie sie üblicherweise über die berufliche Stellung gemessen werden. Diese repräsentieren unterschiedliche Prestigewerte, was wiederum nicht deckungsgleich ist mit Einkommensunterschieden. Es ist nicht immer so, dass die einkommensstärksten Berufe diejenigen sind, die das meiste Prestige haben, und umgekehrt. Und insofern gibt es viele unterschiedliche Messkonzepte für soziale Ungleichheit.

I: Das heißt, soziale Ungleichheit ist nicht nur in einem Bereich messbar, und es gibt verschiedene soziale Ungleichheiten, je nach der Zielvariablen, die man hat?

MD: Richtig. Soziale Ungleichheit ist immer inhärent im Plural zu denken. Es gibt immer verschiedene Dimensionen, die eine Rolle spielen. Wenn man die subjektive Bewertung mit einbezieht, heißt es auch, dass verschiedene Menschen durchaus unterschiedliche Präferenzen haben und unterschiedlich empfindlich sind im Hinblick auf Besser- und Schlechterstellung. Es gibt Leute, die scharf auf Geld sind, und andere sind extrem empfindlich, wenn es um Anerkennung und Prestige geht. Das ist durchaus unterschiedlich.

Eindeutiger ist es im Hinblick auf die Chancenungleichheit. Da geht es darum, ob Angehörige einer bestimmten Bevölkerungskategorie in Relation zu Angehörigen einer anderen Bevölkerungskategorie bessere oder schlechtere Chancen haben, einen höheren Status, höheres Einkommen etc. zu erreichen.

\section{I: Welche Rolle spielt Bildung für soziale Ungleichheit?}

MD: Bildung spielt eine zentrale Rolle. Sie ist im ganzen Aufbau der Ungleichheitsforschung der »Gegenspieler« zur sozialen Herkunft. Moderne Gesellschaften definieren sich sehr stark über den Anspruch, dass das Hineingeborenwerden in eine bestimmte Familie eben nicht das weitere Schicksal von vornherein festlegt, wie das in traditionellen Gesellschaften stärker der Fall war. Aber auch da war es nie absolut der Fall, auch nicht im Mittelalter, wo es doch sehr schwer war, als Bauer ein Adliger zu werden - nahezu unmöglich. Heute sollte es vom Anspruch her nur auf Anstrengung und Fähigkeiten ankommen, wo jemand im Ungleichheitsgefüge später landet. Bildung ist in modernen Gesellschaften der Schlüssel, um diese Anstrengungen und Fähigkeiten abzubilden, die wichtig sind für das Erlangen begehrenswerter Positionen.

I: Wie ausgeprägt ist die soziale Ungleichheit in Deutschland? Wie steht Deutschland in dieser Hinsicht im internationalen Vergleich? 
MD: Im Hinblick auf Ergebnisungleichheit ist Deutschland, wenn wir nicht vergleichbare Gesellschaften wie solche in Afrika außen vor lassen und wir eher sogenannte entwickelte Gesellschaften (die OECD-Welt) betrachten, ein bisschen egalitärer als der Mittelwert. Deutschland ist aber in den letzten 20 Jahren von einer egalitären Gesellschaft in die Mitte gerutscht: Deutschland ist ungleicher geworden. Was Chancenungleichheiten angeht, gibt Deutschland ein durchaus gemischtes Bild ab, zählt aber eher zu den geschlossenen Gesellschaften. Das heißt: Soziale Herkunft ist hier enorm wichtig, auch wenn es in den letzten 30 Jahren große Fortschritte in Richtung Chancenöffnung gegeben hat. Aber die gab es in den anderen Gesellschaften auch. Insbesondere der Hochschulzugang ist davon noch kaum berührt. Ein Studium aufzunehmen und erfolgreich abzuschließen, ist in Deutschland noch stärker herkunftsabhängig als in fast allen Vergleichsländern.

I: Mit welchen Methoden werden die Ursachen sozialer Ungleichheit in der Soziologie typischerweise erforscht?

MD: Es gibt letztlich seit Jahrzehnten zwei etablierte Forschungstraditionen. Das eine ist die Mobilitätsforschung (Kasten 5.1): Da wird der Herkunftsstatus, also die Klassenposition der Eltern, mit der Klassenposition der Kinder verglichen. Dies ergibt relativ komplizierte log-lineare Modelle, wo die tatsächlichen Mobilitätsströme mit Modellen, die bestimmte Chancengleichheitsannahmen repräsentieren, verglichen werden. Hier kann man messen, inwiefern die Realität signifikant von Gleichheitsmodellen abweicht.

\section{Kasten 5.1: Soziale Mobilität}

Soziale Mobilität, hier genauer: intergenerationale soziale Mobilität, vergleicht die soziale Klassenzugehörigkeit der Kinder mit derjenigen ihrer Eltern. Das Ausmaß, in dem die Kinder in eine andere soziale Klasse wechseln als diejenige ihrer Eltern (soziale Herkunft), ist ein Maß für die Offenheit oder Geschlossenheit einer Gesellschaft. Das Ausmaß, in dem es dabei zu Aufstiegen in eine höhere soziale Klasse oder zu Abstiegen in eine niedrigere soziale Klasse kommt, zeigt die Öffnung der Chancenstruktur nach oben oder nach unten. Statistisch kann man herausrechnen, inwiefern Klassenmobilität entweder durch Veränderungen in der Klassenstruktur selbst - etwa einen "Sog« in Richtung Aufwärtsmobilität durch mehr hochqualifizierte Berufe (strukturelle Mobilität) - oder durch Veränderungen in den relativen Chancen der Kinder aus verschiedenen sozialen Klassen (fluide Mobilität) zustande kommt. (Pollak, 2011, S. 173-187)

Die andere Forschungstradition ist die Statuserwerbs- und Lebenslaufforschung (Kasten 5.2), in welcher sehr detailliert die Abfolge von Lebensereignissen und die soziale Herkunft erforscht werden: Was machen Kinder bis zum Schuleintritt, wie gestalten sich Schullaufbahn, Eintritt ins Erwerbsleben und Karriereentwicklung? Es sollen Zusammenhänge von Pfadabhängigkeiten beschrieben werden, also wie früh die Weichen gestellt sind, die, zumindest der statistischen Wahrscheinlichkeit nach, später nicht mehr korrigierbar sind. Deterministisch ist im menschlichen Leben ohnehin kaum etwas. Es wird geschaut, welche Gesellschaften schon sehr früh sehr fixe Weichen haben und welche sehr lange offen sind. Das bezieht sich auf alle Lebensbereiche: die Familie, die Bildung, den Beruf. Da gibt es offenere und geschlossenere 
Systeme. In der neueren Zeit ist die Soziologie viel offener geworden im Hinblick auf das Einbeziehen individueller Entwicklung, was unser gemeinsamer Anknüpfungspunkt ist.

\section{Kasten 5.2: Statuserwerbs- und Lebenslaufforschung}

Werden in der Klassenmobilitätsforschung nur die Ausgangslage (soziale Herkunft) und selbst erreichte Ungleichheitspositionen verglichen, zeichnet sich die Statuserwerbs- und Lebenslaufforschung dadurch aus, dass die verschiedenen dazwischenliegenden Lebensstationen - vor allem die einzelnen Schritte durch das Bildungs- und Beschäftigungssystem, aber auch familiale Ereignisse - je für sich und in ihrem Zusammenhang miteinander untersucht werden. Auf diese Weise kann der Weg von der sozialen Herkunft bis hin zur selbst erreichten Ungleichheitsposition besser dargestellt und erklärt werden. Ein besonderes Interesse gilt dabei der Frage, ob und an welcher "Schaltstelle« genau Weichenstellungen erfolgen und irreversibel werden, d. h. ob und wie sich Bevorzugung und Benachteiligung kumulieren. (DiPrete und Eirich, 2006, S. 271-297)

I: Welche methodischen Herangehensweisen kann die Psychologie zur Erforschung sozialer Ungleichheit beisteuern? (vgl. hierzu auch das Interview mit Prof. Ulrich Trautwein in > Kap. 7)

RR: Zunächst sehe ich keinen prinzipiellen Gegensatz zwischen der Ungleichheitsforschung, wie Herr Diewald sie auffasst, und einer psychologischen Herangehensweise. Wichtig ist, dass wir gleichsam die Kausalketten abarbeiten, das bedeutet, sorgfältig prüfen und nicht voreilig Kausalität unterstellen. Als Psychologen haben wir natürlich einen etwas anderen Fokus. Findet die Soziologie, dass Bildung ganz entscheidend für soziale Ungleichheit ist, dann müssen wir uns als Psychologen fragen: Wie kommt es zu solchen Bildungsunterschieden? Auf diese Weise stellt sich recht schnell die Frage nach der Bedeutung genetischer und aus der Umwelt resultierender Ursachen. Hier ist wichtig, dass wir diese Ursachen trennen müssen und dass wir über die Umwelt nur etwas aussagen können, wenn wir genetisch informierte Designs haben, den Einfluss der Gene also »kontrollieren« können. Bleiben wir bei dem Konstrukt »Bildung « (beispielsweise gemessen an Schulnoten und Qualität des Bildungsabschlusses), dann lässt sich auch gut der Unterschied zwischen Psychologie und Soziologie verdeutlichen: Differenzielle Psychologinnen und Psychologen fragen eher nach der Bedeutung solcher Variablen, deren »Träger« Personen sind. Hier wären neben Intelligenz auch Persönlichkeitsmerkmale und soziale Kompetenzen zu nennen. Weiter ist dann die Frage zu klären, wo die Ursachen für interindividuelle Unterschiede in diesen Variablen liegen. Soziologen nehmen stärker soziale Phänomene in den Blick und würden in diesem Kontext vielleicht Bedingungen für Zugang zu Bildung thematisieren.

Kasten 5.3: Die Logik von Zwillingsforschung und extended-twin-family-Designs Zwillingsforschung (Lieb und Knappe, 2011) ist ein Ansatz, den Einfluss von genetischer Vererbung und Umweltsozialisation voneinander getrennt zu untersuchen. Meist wird die Übereinstimmung bestimmter Merkmale bei Zwillingspaaren beobachtet. Wenn eineiige Zwillinge sich in diesen Merkmalen deutlich ähnlicher sind als zweieiige Zwillinge, wird das als Hinweis auf starke genetische Einflüsse interpretiert: Es wird nämlich davon ausgegangen, dass eineiige und zweieiige Zwillingspaare sich nicht in der Ähnlichkeit ihrer Umwelt 
unterscheiden. Die genetische Ausstattung von eineiigen Zwillingen ist fast identisch, und damit ist die Ähnlichkeit deutlich größer als die zweieiiger Zwillinge. Eine größere Übereinstimmung bei ein- als bei zweieiigen Zwillingspaaren weist also auf genetische Transmission des Merkmals hin.

Die Grundannahme der Zwillingsforschung, dass ein- und zweieiige Zwillingspaare in Umwelten aufwachsen, die in gleichem Ausmaß zur Ähnlichkeit von Zwillingspaaren beitragen (equal environments assumption), ist in vielen Studien geprüft worden (Plomin et al., 2008). So wurde beispielsweise die Ähnlichkeit von falsch klassifizierten Zwillingen (tatsächlich eineiige Zwillinge, die fälschlicherweise als zweieiige angesehen wurden, und tatsächlich zweieiige Zwillinge, die als eineiig diagnostiziert worden waren) verglichen. Es zeigte sich, dass die Ähnlichkeit der Zwillingspaare ihrer tatsächlichen und nicht der vermeintlichen Zygotie entsprach.

Um in Zwillingsstudien auch die gezielte Partnerwahl (assortative mating) zu berücksichtigen, welche die genetische Ähnlichkeit zwischen zweieiigen Zwillingen erhöhen kann und dann fälschlicherweise als Effekt der Umwelt interpretiert wird, wurde in den letzten Jahren vermehrt das extended-twin-family-Design angewandt. Dabei werden neben den Zwillingen selbst auch deren Eltern, eventuell vorhandene Geschwister, Großeltern, Onkel, Tanten und viele weitere genetisch verwandte Personen in die Studie mit einbezogen. Dieses Vorgehen erlaubt es, neben den statistisch genaueren Bestimmungen von Anlage- und Umwelteinflüssen (im Vergleich zu derselben Zahl von Zwillingen ohne Daten der Familienangehörigen) Effekte des Aufwachsens als Zwillingspaar zu kontrollieren. (Lieb und Knappe, 2011, S. 91-106)

I: Bitte erläutern Sie uns die Logik von genetisch informierten Studien, z. B. Zwillingsstudien (Kasten 5.3)!

RR: Die Logik ist im Grunde recht einfach. Menschen unterscheiden sich biologisch gesehen in ihrer genetischen Ausstattung, das heißt, fast jeder Mensch hat ein einzigartiges Genom. Tatsächlich sind es eher wenige Gene, in denen Menschen sich unterscheiden, aber schon wenn wir nur auf das Äußere von Menschen schauen, wird deutlich, dass die zahlenmäßig eher geringen genetischen Differenzen doch sehr bedeutsam sind.

Für die Untersuchung psychischer Merkmale sind quantitativ-genetische Studien ein ganz wichtiger Weg, um herauszufinden, wie bedeutsam Anlagen und Umweltbedingungen sind. Ein Beispiel für diese Forschung stellen Zwillingsstudien dar: Im einfachsten Fall würde man eine Stichprobe eineiiger Zwillinge untersuchen, die sehr früh nach der Geburt getrennt wurden und dann in zufällig zugewiesenen Umwelten aufgewachsen sind. In diesem Fall kann die Ähnlichkeit zwischen solchen Zwillingen ausschließlich auf genetische Effekte zurückgeführt werden.

In unseren eigenen Studien untersuchen wir zusammen aufgewachsene Zwillinge. In diesem Fall wird auf genetische Effekte geschlossen, wenn eineiige Zwillinge bei gleicher Ähnlichkeit der Umweltbedingungen einander ähnlicher sind als zweieiige. Dies ist ein etwas schwächeres Vorgehen als die Untersuchung getrennt aufgewachsener Zwillinge. Daher sind Verhaltensgenetiker bestrebt, Informationen aus verschiedenen Familienbeziehungen (einschließlich Adoptivfamilien) zu kombinieren. Herr Diewald und ich planen gerade eine Studie, in der wir ein sogenanntes extended-twin-family-Design verwenden, in dem wir neben den ein- und zweieiigen Zwillingen auch deren Eltern und eventuell vorhandene weitere Geschwister mit einbeziehen. Aus den vielfältigen Beziehungen (Eltern - Kind, Zwillinge untereinander, Zwillinge - "normale« Geschwister) lassen sich dann mittels entsprechender Analyseverfahren Anlage- und Umwelteinflüsse auf psychische Merkmale sehr genau untersuchen. 
I: Häufig wird die Tatsache, dass etwas durch Gene beeinflusst wird, als Beleg dafür gesehen, dass etwas nicht beeinflussbar, also unveränderbar ist. Stimmt diese Annahme?

RR: Diese Frage lässt sich nicht einfach mit ja oder nein beantworten. Zunächst müssen verschiedene Arten der Veränderung unterschieden werden. Verhaltensgenetik beschäftigt sich mit Unterschieden zwischen Menschen, das bedeutet zugleich, dass sie keine Aussagen über gesellschaftliche oder kulturumfassende Prozesse macht. Dies lässt sich gut am Beispiel der Intelligenz verdeutlichen. James R. Flynn untersuchte im letzten Jahrhundert Veränderungen im Mittelwert in IQ-Werten. Ihm war aufgefallen, dass mit der Neunormierung von Intelligenztests die Normen stets etwas »strenger « wurden (für denselben IQ-Wert müssen im Test etwas mehr Aufgaben gelöst werden), und fand bei einer systematischen Analyse, dass die Testleistungen der Menschen in modernen Industriestaaten alle drei Jahre um zirka einen IQ-Punkt angestiegen waren. Solche Mittelwertveränderungen sind auch dann möglich, wenn ein Merkmal eine starke genetische Beeinflussung aufweist. ( $v g l$. hierzu auch das Interview mit Prof. Elsbeth Stern in $>$ Kap. 6)

Eine andere Frage ist jedoch, ob sich auch die Rangreihen von Personen verändern lassen, wenn das Merkmal eine hohe Erblichkeit aufweist. Ist es also möglich, dass aus schlechten Schülern durch geeignete Förderung bessere (auch im Vergleich mit den Mitschülern) Schüler werden? Auf derartige Rangreihen und Vergleiche zwischen Menschen bezieht sich der Begriff der Erblichkeit direkt. Auch hier ist aber die Antwort: Prinzipiell ist dies möglich. Wenn wir die Erblichkeit eines Merkmals untersuchen, dann gilt die resultierende Aussage (z. B. $60 \%$ genetischer Einfluss) in der spezifischen soziohistorischen Situation, in der die Untersuchung gemacht wurde. Sie ist auf die zu einem bestimmten Zeitpunkt in einer Gesellschaft real zu findenden und mit bestimmten Häufigkeiten zu findenden Umweltbedingungen bezogen.

Für viele im Bildungsbereich bedeutsame Merkmale sind solche gezielten, häufig auf leistungsschwächere Personen gerichteten Maßnahmen sehr aufwendig und waren nicht immer von Erfolg gekrönt. Wichtig ist selbstverständlich, auch das Zusammenspiel zwischen Anlagen und Umwelten zu beachten, denn in der Regel finden wir bedeutsame Zusammenhänge zwischen beiden.

Wir merken an allen Beispielen, auch aus dem Kontext der Bildungsforschung, dass es sehr häufig ein Zusammenspiel aus Anlage und Umwelt gibt. Ein ganz einfaches Bespiel: In vielen Industriestaaten ist im Bildungsbereich eine Anlage-Umwelt-Kovariation eingeplant. Das heißt, diejenigen Menschen werden stärker gefördert (z. B. sie besuchen länger eine Schule), die bessere Fähigkeiten haben, und diejenigen, die die geringsten Fähigkeiten haben, werden weniger trainiert, gefördert und gebildet. Das ist offenbar so gewollt und sicher auch ökonomisch. In der Konsequenz werden die vorhandenen genetischen Unterschiede in bildungsrelevanten Fähigkeiten und Merkmalen weiter durch selektive Förderung und Anreize verstärkt.

MD: Aber man kann kompensatorische Strategien fahren, was naheliegt, wenn es beispielsweise einen größeren Bedarf an hochqualifizierten Personen gibt, als man sie mit den üblichen ökonomischen Verfahren im Bildungssystem produzieren könnte. Man braucht mehr Leute und muss mehr investieren.

RR: Ja, das ist möglich, aber es gibt sicher auch Grenzen, da sind wir uns einig. Es gilt nicht das Skinner'sche Ideal, dass wir aus jedem Menschen durch geeignete Verstärkungspläne alles machen können. 
I: Welche neuen Erkenntnisse und welchen Nutzen erwarten Sie von der Durchführung genetisch informierter Studien für die Forschung zur sozialen Ungleichheit?

RR: Es gibt nur wenige Studien zu genetischen Ursachen sozialer Ungleichheit, und diese werden schnell überinterpretiert. Sie werden publikumswirksam aufbereitet, und dass dabei charakteristische Fehler gemacht werden, haben wir bereits bei der »Bell Curve« (Herrnstein und Murray, 1994) und auch bei Sarrazin (2010) gesehen. Das ist nicht nur langweilig, sondern führt zu nichts. Am Ende wird die Frage selbst diskreditiert: Es ist ja schon nahezu anrüchig zu fragen, ob es genetische Ursachen für soziale Ungleichheit gibt. Dabei ist es naheliegend, dass nicht jeder jeden Bildungsabschluss erreichen kann, auch nicht in einem stark auf Kompensation und individuelle Förderung ausgerichteten System, was wir gegenwärtig nicht haben. ( $v g l$. hierzu auch das Interview mit Prof. Elsbeth Stern in > Kap. 6)

I: Prof. Diewald, für viele Ihrer Kollegen dürfte es befremdlich sein, dass die Soziologie in ihren Studien nun auch Gene berücksichtigen soll. Stoßen Sie mit Ihrem Ansatz auf Unverständnis oder gar Abwehr?

MD: Ja, immer noch. Das ist gar nicht anders zu erwarten. Das Thema war jahrzehntelang hart tabuisiert. Man hat sich nicht nur nicht dafür interessiert, sondern es gab eine dezidierte Abwehr, und die ist nicht über Nacht verschwunden. Trotzdem würde ich sagen, dass sich die Lage in den letzten zehn Jahren deutlich geändert hat. Ich habe einen Artikel in der Zeitschrift für Soziologie publiziert (Diewald, 2010b), der hatte in den ersten anderthalb Jahren die meisten Aufrufe, was niemand erwartet hat, nicht einmal die Zeitschrift für Soziologie selbst. Der Artikel ist mit weitem Abstand am meisten heruntergeladen worden. Das zeigt, dass einiges ins Rollen geraten ist, was mich überrascht hat. Das Interesse und die Akzeptanz sind zweifelsohne gestiegen, in Teilen ist das Thema aber noch tabuisiert.

\section{I: Auf welche Herausforderungen stoßen Sie bei der transdisziplinären Zusammenarbeit?}

RR: Meine Erfahrung ist, dass die Beteiligten an interdisziplinärer Forschung zunächst eine gemeinsame Sprache finden müssen. Am Anfang versteht man einander einfach erst einmal nicht. Dass wir beide uns relativ gut verständigen können, ist auch darin begründet, dass Herr Diewald schon seit vielen Jahren mit Psychologen zusammenarbeitet. Außerdem habe ich einige Jahre Soziologie studiert. Dadurch ergibt sich eine gewisse Nähe. Meine Erfahrung ist, dass es lange braucht, um die Sprache des anderen zu verstehen, um herauszufinden, was dem anderen wichtig ist. Bei gemeinsamen Projekten reicht es ja nicht, den groben Sinn des Gesagten oder Geschriebenen zu verstehen. Es kommt darauf an, genau zu begreifen, was dem Gegenüber wichtig ist, und auch die Standards und Konventionen der beteiligten Disziplinen zu berücksichtigen. Solche »Fachbefindlichkeiten« sind gelegentlich sehr unterschiedlich.

MD: Anders herum ist das genauso. Ich habe schon viele Jahre Erfahrung damit. Es begann bereits mit meiner Diplomarbeit, die ich über soziale Netzwerke geschrieben habe. Das ist ein Feld, in dem über die coping- und social-support-Theorien eine enge Verschränkung von Soziologie und Psychologie stattfindet. Hier gibt es ein hohes Maß an wechselseitiger Wahrnehmung der jeweiligen Forschung, und man wird langsam sozialisiert, sich auch mit den anderen Sichtweisen auseinanderzusetzen. Und sie sind tatsächlich unterschiedlich: Psychologen legen auf andere Dinge Wert in Theorie und Empirie. Da gibt es ganz klare Unterschiede darin, was man 
wertschätzt. Sogar die Art, wie man einen Zeitschriftenaufsatz aufbaut, folgt unterschiedlichen Regeln in der Soziologie und Psychologie. Das muss man lernen.

Ich komme aus der Lebenslaufforschung. Da gibt es das Spezifikum, dass die deutsche Lebenslaufforschung sich immer hart gegen die Psychologie abgegrenzt hat. Im angelsächsischen Raum ist das ganz anders, life course ist dort sowohl in der Psychologie als auch in der Soziologie ein gleichermaßen verwendeter Begriff. Er ist von vornherein viel interdisziplinärer angelegt. Personen wie Glen Elder sind sowohl in der Soziologie als auch der Psychologie als maßgeblich anerkannt. Das war in der deutschen Soziologie nicht so. Damals am MaxPlanck-Institut für Bildungsforschung haben Jutta Heckhausen, Johannes Huinink und ich in die soziologischen Lebenslaufuntersuchungen psychologische Konstrukte, nämlich Kontrollüberzeugungen und Kontrollstrategien, eingebaut. Ich wurde teilweise als Verräter an der Profession beschimpft. Das war in den 1990er Jahren. Das ist lange her und nun definitiv nicht mehr so. Es gibt Großprojekte, in denen Psychologen und Soziologen eng zusammenarbeiten.

I: Das heißt, Sie sind der Überzeugung, dass die Zusammenarbeit mit Psychologen und Verhaltensgenetikern für die Soziologie eine Bereicherung darstellt.

MD: Unbedingt. Das habe ich nachdrücklich angestrebt. Nun hoffe ich, dass es sich bewahrheitet.

I: Wir haben darüber gesprochen, welchen Beitrag psychologische Methoden für die Erforschung soziologischer Fragestellungen leisten können. Welche Beiträge kann die Soziologie zu psychologischen Fragestellungen leisten?

RR: An sich ist das einfach zu beantworten: Es geht darum, sozial relevante Maße (z. B. Indikatoren sozialer Ungleichheit) vorherzusagen und zu erklären. Mich interessiert, warum Menschen sehr unterschiedlich in ihren Lebensergebnissen sind. Warum ist jemand erfolgreich, reich, schön und ein anderer nicht? Das ist doch eine spannende Frage! Derartige Fragestellungen sind einfach zu erklären, weil sie fast jeder versteht und selbst spannend findet. Bei solchen Fragen kommt man nicht umhin, Soziologen zu befragen. Wie Sie gehört haben, gibt es spezielle Strukturen, wie man soziale Ungleichheit begreift. Da profitiert die Psychologie von der Zusammenarbeit. Die Kernfrage an sich bleibt für mich eine differenziell-psychologische, und es ist sehr gut zu wissen, dass meine eigene begrenzte Sichtweise durch andere Herangehensweisen ergänzt wird.

I: Werfen wir einen Blick in die Zukunft: Wo würden Sie gern mit Ihrer Forschung in fünf Jahren stehen?

MD: In fünf Jahren haben wir erfolgreich die Studie etabliert und sehen, dass es sich gelohnt hat. Dann sollten wir gute Erträge haben.

I: Ihre Forschung trägt zum grundlegenden Verständnis der Ursachen sozialer Ungleichheit bei. Werden sich daraus auch praktische Schlussfolgerungen ableiten lassen, z. B. für Bildungsprozesse?

RR: Das ist nicht leicht zu beantworten, denn wenn wir alle Ergebnisse bereits kennen würden, dann müssten wir die Untersuchung nicht mehr machen. Perspektivisch wird unsere Zusammenarbeit auch praktische Bedeutung haben, aber nicht in der Art, dass wir konkrete 
Vorschläge für die Schulpolitik prüfen. Wir wollen möglichst genau betrachten, wie die Anlagen von jungen Menschen mit Elternhaus, Schule, Ausbildung und anderen Umwelten zusammenwirken. Je besser wir dies verstehen, um so eher können wir konkrete Maßnahmen in diesen Bereichen ableiten und begründen. Davon auszugehen, wir könnten in fünf Jahren Politik beraten, halte ich für zu optimistisch.

MD: Die meisten Einflüsse in die Politik kommen eher unbeabsichtigt zustande. Weil Ergebnisse nicht vorhersehbar sind, kann man Einflussnahme nicht planen. Ergebnisse machen Forschung wirkmächtig; die PISA-Studie ist ein Beispiel dafür. Niemand hat vorher erwartet, dass diese Studie sich so stark auf die Politik niederschlägt. (vgl. hierzu auch das Interview mit Prof. Hans Anand Pant und Prof. Petra Stanat in > Kap. 2)

Umgekehrt sind andere Projekte wie der Sonderforschungsbereich 3 (»Mikroanalytische Grundlagen der Gesellschaftspolitik«), welcher akademisch sicher einer der ertragreichsten Sonderforschungsbereiche ist, die es in den Sozialwissenschaften gegeben hat, in ihrem Anspruch, direkt in die Politik hinein zu wirken, eher enttäuscht worden.

Bei unserer Studie könnte man nun herausfinden, auf welche Art und Weise bestimmte Personen - auf verschiedenen Ebenen wie Persönlichkeitskonstrukten und genetischer Veranlagung differenziert - in unterschiedlichen Situationen unterschiedlich erfolgreich sind. Es soll von dem einfachen linearen Erfolgsdenken weggekommen werden: Man schraubt an einer Stelle, und es geht für alle bergauf oder bergab. Stattdessen passt für unterschiedliche Menschen die eine oder die andere Maßnahme unterschiedlich gut.

Gerade in der Soziologie schneiden statistische Modelle wie die Varianzaufklärung eher schwach ab, was damit zu tun hat, dass für unterschiedliche Personen gleiche Situationen nicht das Gleiche bedeuten. Dies wurde in der Vergangenheit unzureichend berücksichtigt. Mit unserer Untersuchung kommen wir sicher einige Schritte weiter. Mit der Berücksichtigung genetischer Variation als Ausgangspunkt kann man der Frage auf den Grund gehen, was Gesellschaften aus ihren Mitgliedern machen - von Beginn an: Je nach genetischer Ausstattung können Individuen von bestimmten Umwelten mehr oder weniger profitieren.

RR: Wir erwarten, dass die Wirkung bzw. Bedeutsamkeit von Genen für unterschiedliche Gruppen unterschiedlich stark ausfällt. Wir haben Befunde aus der Intelligenzforschung, dass bei Menschen, die in ökonomisch extrem benachteiligten Umwelten aufwachsen, genetische Effekte nicht so deutlich sind wie in Mittelstands- bis Oberschichtsumwelten (Kasten 5.4). Bei diesen spielen genetische Unterschiede eine stärkere Rolle. So etwas ist wichtig zu sehen, um praktische Konsequenzen ziehen zu können. Man könnte Ressourcen auf bestimmte Förderprogramme lenken, statt undifferenziert »mit der Gießkanne« vorzugehen.

\section{Kasten 5.4: Anlage-Umwelt-Interaktion}

Wenn sich genetische Dispositionen in unterschiedlichen Umwelten unterschiedlich stark entfalten, sprechen wir von Anlage-Umwelt-Interaktionen. Diese Interaktionen wurden für die Entwicklung der Intelligenz in der Kindheit nachgewiesen. In mehreren Studien zeigte sich, dass Intelligenz in einer Gruppe von Kindern, deren Eltern einen niedrigen sozioökonomischen Status haben, weniger stark genetisch beeinflusst ist als in einer Gruppe mit höherem sozialen Status der Eltern. Anstelle der genetischen Effekte sind in den statusniedrigen Gruppen Effekte der Umwelt von größerer Bedeutung. Traditionell wird hier unterschieden 


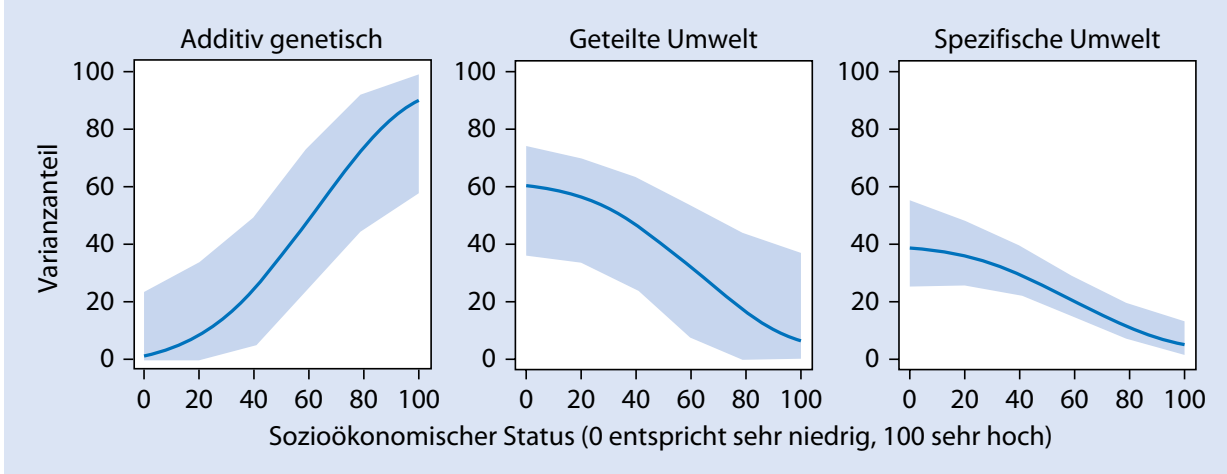

- Abb. 5.1 Sozioökonomischer Status und Intelligenzentwicklung bei Kindern im Alter von sieben Jahren. $0=$ sehr niedrig, $100=$ sehr hoch. (Adaptiert nach Turkheimer et al., 2003, S. 627)

zwischen Effekten der von den Geschwistern geteilten und der für jedes Geschwisterkind spezifischen Umwelt. Die Ergebnisse einer Studie von Turkheimer und Kollegen (2003) sind in - Abb. 5.1 zusammengefasst. Es zeigt sich, dass mit steigendem sozioökonomischen Status die genetische Beeinflussung stark zunimmt (links: von nahezu $0 \%$ bis auf über $80 \%$ ), während die Bedeutung der Umwelt (Mitte und rechts) entsprechend abnimmt. Diese Befunde stützen die Auffassung, dass die zugrunde liegende genetische Disposition erst dann zur Ausbildung von Intelligenz führt, wenn die Umwelt entsprechende Entwicklungsbedingungen bereitstellt.

I: Herr Diewald, Herr Riemann, wir danken Ihnen für das Gespräch!

Video des Interviews:

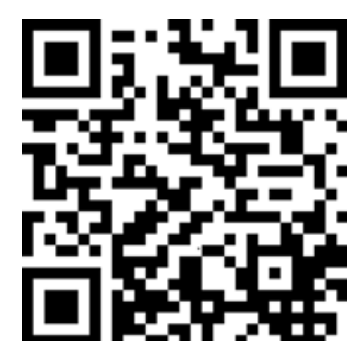

\section{http://tinyurl.com/DiewRiem}

\section{Zitierte und weiterführende Literatur}

Diewald, M. (2010a). Ungleiche Verteilungen und ungleiche Chancen. Zur Entwicklung sozialer Ungleichheiten in der Bundesrepublik. In F. Faulbaum \& C. Wolf (Hrsg.), Gesellschaftliche Entwicklungen im Spiegel der empirischen Sozialforschung. Schriftenreihe der ASI-Arbeitsgemeinschaft Sozialwissenschaftlicher Institute (S. 11-38). Wiesbaden: VS Verlag für Sozialwissenschaften. 
Diewald, M. (2010b). Zur Bedeutung genetischer Variation für die soziologische Ungleichheitsforschung. Zeitschrift für Soziologie, 39, 4-21.

Diewald, M., \& Mayer, K. U. (2009). The sociology of the life course and life span psychology: Integrated paradigm or complementing pathways? In A. de Ribaupierre, D. Joye \& M. Oris (Hrsg.), Advances in Life Course research. Special Issue: Reconsidering the Linked Life Principle (S. 5-14). Amsterdam: Elsevier.

DiPrete, T. A., \& Eirich, G. M. (2006). Cumulative advantage as mechanism for inequality: A review of theoretical and empirical developments. Annual Review of Sociology, 32, 271-297.

Herrnstein, R. J., \& Murray, C. (1994). The bell curve. Intelligence and class structure in American Life. New York: Free Press.

Lieb, R., \& Knappe, S. (2011). Familiäre Transmission psychischer Störungen. In H.-U. Wittchen \& J. Hoyer (Hrsg.), Klinische Psychologie \& Psychotherapie (2. Aufl., S. 91-106). Heidelberg: Springer.

Plomin, R., DeFries, J. C., McClearn, G. E., \& McGuffin, P. (2008). Behavioral genetics. New York: Worth.

Pollak, R. (2011). Soziale Mobilität. In Statistisches Bundesamt (Hrsg.), Datenreport 2011 (S. 180-187). > https:// www.destatis.de/DE/Publikationen/Datenreport/Downloads/Datenreport2011.pdf?_blob=publicationFile. Zugegriffen: 25. Feb. 2013.

Riemann, R., \& Spinath, F. (2005). Genetik und Persönlichkeit. In P. Netter \& J. Henning (Hrsg.), Biopsychologische Grundlagen der Persönlichkeit (S. 539-628). Heidelberg: Springer

Rowe, D. C. (1997). Genetik und Sozialisation: Die Grenzen der Erziehung. Weinheim: Psychologie Verlags Union.

Sarrazin, T. (2010). Deutschland schafft sich ab. Wie wir unser Land aufs Spiel setzen. München: DVA.

Turkheimer, E., Haley, A., Waldron, M., D’Onofrio, B. M., \& Gottesman, I. I. (2003). Socieoeconomic status modifies heritability of IQ in young children. Psychological Science, 14, 623-628. 